\title{
The unusual multi-wavelength SED of two optical dropout galaxies
}

\author{
Daniel Schaerer ${ }^{1,2}$, Frederic Boone ${ }^{2}$ and Nicolas Laporte ${ }^{2}$ \\ ${ }^{1}$ Observatoire de Genève, Université de Genève, 51 Ch. des Maillettes, 1290 Versoix, \\ Switzerland \\ ${ }^{2}$ CNRS, IRAP, 14 Avenue E. Belin, 31400 Toulouse, France \\ email: daniel.schaerer@unige.ch
}

\begin{abstract}
We have used deep optical, near-IR, and IR observations from VLT, Spitzer, Herschel, and LABOCA in strong lensing clusters to study distant galaxies. In searches for optical-dropout galaxies (i.e. for $z \gtrsim 7$ candidates) we have found several galaxies with very unusual SEDs characterised by a strong spectral break, presumably indicative of high- $z$, although the objects are detected even in the Herschel bands between 160 and $500 \mu \mathrm{m}$ and at $870 \mu \mathrm{m}$. The latter indicates, from simple estimates of the bolometric luminosity and from the IR SED, that these ob jects are most likely at $z \sim 2-2.5$.

The resulting SEDs imply very high IR/UV ratios, indicative of very large attenuation. Despite this, the large spectral break observed between the optical and near-IR data is difficult to understand with currently know spectral templates from galaxies, EROs, SMGs, and others, both empirical and theoretical ones.
\end{abstract}

Keywords. galaxies: starburst, galaxies: high-redshift, galaxies: evolution, galaxies: formation

\section{Introduction}

Recently we have identified ten $z>7$ candidates in the field of the cluster Abell 2667 using photometric dropout criteria based on deep observations with HAWK-I on the ESO VLT (Laporte et al. 2011). Two galaxies of this sample were later found to be clearly detected in the far-IR with Herschel and one of them with LABOCA (at 870 $\mu \mathrm{m})$, as described in Boone et al. (2011). Most likely, this indicates that these galaxies are "low" redshift $(z \sim 2-2.5)$ interlopers. However, their SED including a well-defined spectral break appears quite unusual, and extreme. The origin of the spectral break and the nature of these objects remains puzzling, as we briefly describe here. For more details see Boone et al. (2011).

\section{Observed SEDs of $z-$ and Y-dropout galaxies}

Targetting the strong lensing cluster Abell 2667 we have obtained deep optical imaging (I, z with FORS2/VLT), deep near-IR data in the Y, J, H, and Ks bands with HAWKI/VLT. For the cluster, which is part of our ongoing Herschel Open Time Key project, the "Herschel Lensing Survey" (Egami et al. 2010), we also have data from Spitzer (from 3.6 to $8 \mu \mathrm{m}$ ), MIPS $24 \mathrm{~m}$, LABOCA $870 \mu \mathrm{m}$, and the VLA (1.4 GHz) (see Boone et al. 2011). Our galaxies are selected from $\mathrm{H}+\mathrm{K}$ images after applying $\mathrm{z}$ - and $\mathrm{Y}$-band dropout criteria (i.e. Lyman break selection + visual inspection). All 10 optical dropout candidates found in this field are discussed in Laporte et al. (2011). Two of them, denoted z1 and Y5, are detected at $24 \mu \mathrm{m}$, and/or in several Herschel bands. The SED of z1 is shown in Fig. 1. The near-IR magnitudes of the two ob jects is $23.5-24$ ( $\mathrm{H}$ to $\mathrm{K}$ band 

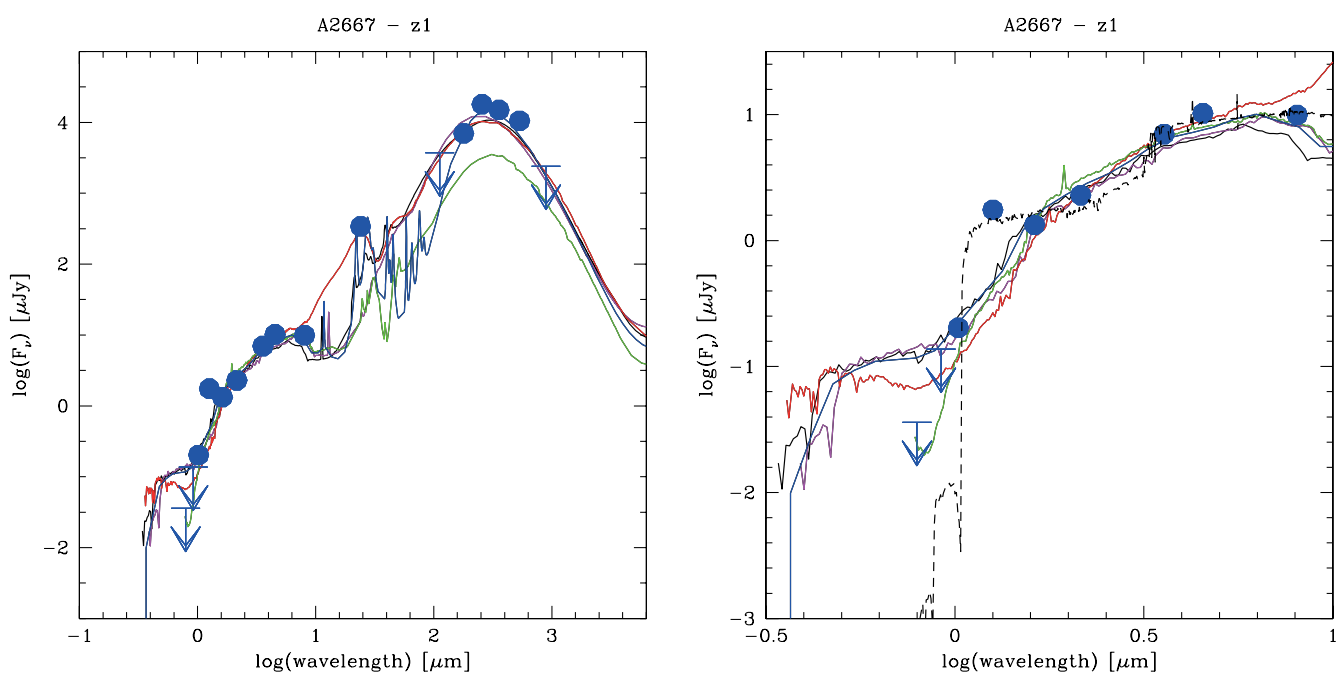

Figure 1. Best-fit SEDs for z1 using different templates: Chary \& Elbaz (2001), Polletta et al. (2007), and Michalowski et al. (2010). Left: global SED, Right: zoom on region with the spectral break. Figure from Boone et al. (2011)

in $\mathrm{AB}$ ). This would imply very luminous objects, if at $z>7$ ! Still high-z is formally favoured for z1 from photometry redshift including luminosity prior (cf. Laporte et al.) The IR SED would imply a very high $L_{\mathrm{IR}} \sim(0.5-1.5) \times 10^{14} \mathrm{~L}_{\odot}$, and would requite a very high dust temperature $(>100-130 \mathrm{~K})$ if at high $\mathrm{z}$, which are both very unlikely. The overall multi-wavelength SED indicates best-fit redshifts at $z \sim 2-2.5$. Then one has $L_{\mathrm{IR}} \sim(2-3) \times 10^{12} \mathrm{~L}_{\odot}$, corresponding to $\mathrm{SFR} \sim 400-500 \mathrm{M}_{\odot} \mathrm{yr}^{-1}$.

The galaxies exhibit a very large flux ratio between IR and rest-UV (comparable to extreme sub-mm galaxies). The observed spectral break (used as selection criterium) is stronger than predicted by current empirical and theoretical spectral templates, as shown in Fig. 1. The origin of such a strong spectral break is unknown. Extinction, emission lines, and multiple populations appear difficult to explain it (see Boone et al. 2011). Our two galaxies may be comparable to other extreme objects, such as the sub-mm galaxy GN10=GOODS 850-5 or others (cf. Wang et al. 2009, Daddi et al. 2009, Huang et al. 2011). How frequent such possible contaminant are in high-z searches remains to be seen.

This work received support from the Agence Nationale de la Recherche bearing the reference ANR-09-BLAN-0234.

\section{References}

Boone, F., Schaerer, D., et al. 2011, A\& A, 534, A124

Chary, R. \& Elbaz, D. 2001, ApJ, 556, 562

Daddi, E., Dannerbauer, H., Krips, M., et al. 2009, ApJ,, 695, L176

Egami, E., et al. 2010, A\& A, 518, L12

Huang, J.-S., et al. 2011, ApJ, 742, L13

Laporte, N., Pelló, R., Schaerer, D., et al. 2011, A\&A, 531, A74

Michałowski, M. J., Watson, D., \& Hjorth, J. 2010, ApJ, 712, 942

Polletta, M., Tajer, M., Maraschi, L., et al. 2007, ApJ, 663, 81

Wang, W., Barger, A. J., \& Cowie, L. L. 2009, ApJ,, 690, 319 